\title{
Development and Characterization of Sisal Fiber Reinforced Polypropylene Composite Materials
}

\author{
A. G. GEREZGIHER ${ }^{1}$, H. A. BSRAT ${ }^{1,2}$, A. SIMON ${ }^{1}$, T. SZABO ${ }^{1}$ \\ 1University of Miskolc, faculty of materials science and engineering, gebresas@gmail.com, femandi@uni- \\ miskolc.hu, polsztam@uni-miskolc.hu \\ ${ }^{2}$ Adi-Grat University, halefom.aregay@yahoo.com
}

\begin{abstract}
In most of the developing countries, plastic polypropylene is not fully recycled and converted in-to use after it is once used. Sisal fiber is also widely available in different developing countries like Ethiopia. Adding this two materials and developing automotive interior part was taken as a primary motive for it reduces cost and is environmentally friendly. Thus, the main purpose of this research is to develop composite material from natural fibre (sisal fiber) reinforced with recycled plastic waste (polypropylene) for interior automobile accessories specifically for internal door trim panel application. This research examines effect of fiber length, fiber loading and chemical treatment of fiber on the physical and chemical properties of the sisal fiber reinforced polypropylene (SFRPP) composite material. The waste polypropylene and the treated and untreated sisal fiber with variable length and weight ratio (fiber/matrix ratio) were mixed. Flammability of sisal fiber reinforced Polypropylene (SFRPP) composites material was examined by a horizontal burning test according to ASTM D635 and chemical resistance of the sisal fibre reinforced PP composites was studied using ASTM D543 testing method. The result on the flammability test shows that treated fiber has lower burning rate than untreated fiber and decreases with increase in fiber length and fiber loading. The resistance of the composites to water has increased as the fiber length increases and decreased as the fiber loading increase. Generally, SFRPP composite is found to have better resistance to water than $\mathrm{NaOH}$ and $\mathrm{H}_{2} \mathrm{SO}_{4}$ and treating the fiber has brought considerable improvement on chemical resistance of the composite. Fiber loading and fiber length has positive and negative effect on the flammability of the SFRPP composite respectively.
\end{abstract}

Keywords: Sisal fibre, Polypropylene, compressive moulding, flammability, chemical resistance

\section{Introduction}

There has been a growing interest in utilizing natural fibers as reinforcement in polymer composite for making low cost construction, packaging, automobile parts, biomedical and electronic materials in recent years [1]. Fibers of this type, for instance, hemp and flax, are successfully used as packaging material, interior panels in vehicles and building components among others. Also, natural fibers like banana, sisal, hemp and flax, jute, coconut, bamboo, sponges, wood dusts and oil palm [2-8] have attracted scientists and technologists for applications in consumer goods, low-cost housing and other civil structures. A number of investigations have also been conducted on several types of natural fibers to study the effect of these fibers on the physical, chemical and mechanical properties of composite 
materials [9]. So many researches have proved natural fibers to be effective reinforcement as simple fillers in thermoplastic and thermo setting matrix composites for automotive sectors. Jute fabric reinforced polyester composites were also tested for the evaluation of mechanical properties and compared with wood composite and it was found that the jute fibre composite has better strengths than wood composites [10]. Fiber reinforced composite materials have been widely used in various transportation vehicle structures because of their high specific strength, modulus and high damping capability. If composite materials are applied to vehicles, it is expected that not only the weight of the vehicle is decreased but also the noise and vibration are reduced. In addition, composites have a very high resistance to fatigue and corrosion [12-13]. On the other hand, synthetic fiber has low fiber/matrix adhesion, fluctuations in fiber properties, low fire resistance and also cause Environmental pollution during the disposal of the used composite materials [16, 17].

Thus, this research is initiated with the objective to develop sisal fiber reinforced waste polypropylene (SFRPP) composite materials for internal door trim application; and specifically investigate and improve the chemical resistance and flammability property of sisal fiber reinforced waste polypropylene composite.

\section{Material and Experimental Procedure}

\subsection{Sisal fiber}

For this research work, sisal plant leaves was collected from northern Ethiopia cutting at their base from the harvest. The fibers are extracted through hand extraction technique with the help of metallic knife. Initially the leaves were trimmed in a longitudinal direction into strips for ease of fiber extraction. The peel is clamped between wood plank and knife; and hand-pulled in a longitudinal direction gently, removing the resinous material. Then the extracted fiber was washed with pure water in order to get a clean sisal fiber. Subsequently, the extracted fibers are sun-dried and are ready for fabrication.

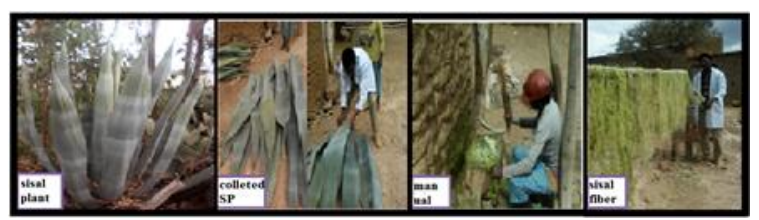

Figure 1. Sisal fiber preparation (Manual peel technique)

\subsection{Fibre treatment and preparation}

Alkali treatment is the simplest method of chemical treatment of fibers; it leads to an increase in the amount of amorphous cellulose at the expense of crystalline cellulose. The important modification occurring here is the removal of hydrogen bonding in the network structure. The following reaction takes place as a result of alkali treatment [23].

Fiber- $\mathrm{OH}+\mathrm{NaOH} \rightarrow$ Fiber- $-\mathrm{O}^{-} \mathrm{Na}^{+}+\mathrm{H}_{2} \mathrm{O}$ .Eq [1] 
Up on the addition of alkali to cellulose fiber, there is a swelling reaction which relaxes the natural crystal structure of the cellulose. The degree of swelling and lattice transformation into cellulose-II is highly influenced by the type and concentration of alkali chemicals [6]. $\mathrm{Na}+\mathrm{O} \mathrm{NaOH}$ alkali has got a favourable diameter compared to other alkali chemicals, which is able to widen the smallest pores in between the lattice planes and penetrate into them. As a result higher amount of swelling is found from sodium hydroxide treatment. Consequently, new Na-cellulose-I lattice, a lattice with relatively large distances between the cellulose molecules is formed and water is filled in the spaces with in the molecules. In this structure, the OH-groups of the cellulose are converted into $\mathrm{O}$ Na-groups, expanding the dimensions of molecules. Subsequent rinsing with water will remove the linked Na-ions and convert the cellulose to a new crystalline structure, i.e. cellulose-II, which is thermodynamically more stable than cellulose-I. Sodium hydroxide can cause a complete lattice transformation from cellulose-I to cellulose-II, in contrast to other alkalis that produce only partial lattice transformation [24].

In this study, a $4 \% \mathrm{NaOH}$ solution was used to treat the raw Sisal fibers, in order to modify their fiber structures.

During this process dried and chopped sisal fibers were placed in a tray, then $4 \% \mathrm{NaOH}$ solution was added. The fibers were soaked for 24 hours to remove fatty impurities. The fibers were then washed thoroughly with water to remove the excess of $\mathrm{NaOH}$ sticking to the fibers [27]. Lastly, the fibers were allowed to dry in sun light for 3 days.

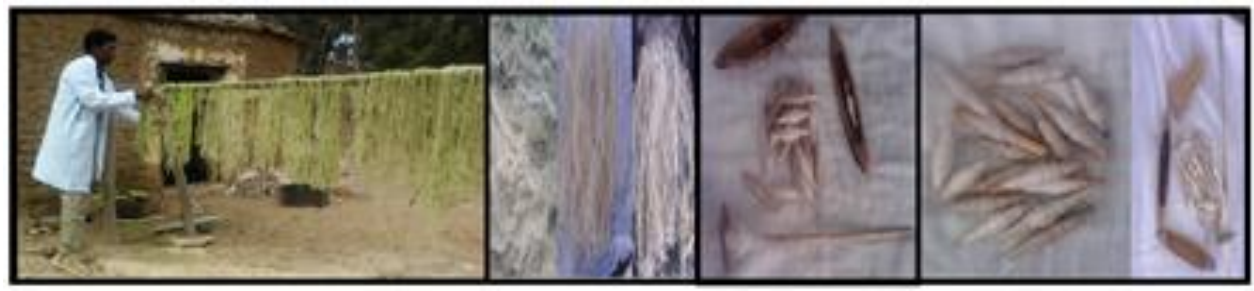

Figure 2. Extracted Sisal fiber

The fibers were cut into $\sim 3 \mathrm{~mm}, 6 \mathrm{~mm}, 9 \mathrm{~mm}$, and $12 \mathrm{~mm}$ using a pair of scissors and made ready for mixing as shown below.

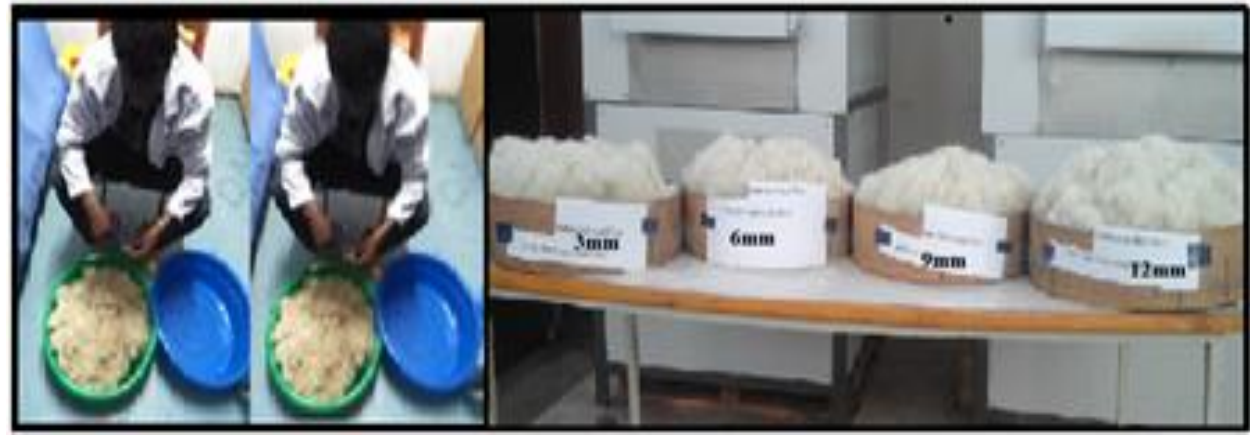

Figure 3. Chopped sisal fiber

\subsection{Polymer matrix}

The recycled Polypropylene matrix material was collected from broken chairs and tables of nearby cafeterias and hotels with print information of Polypropylene grade-5 written on them. 


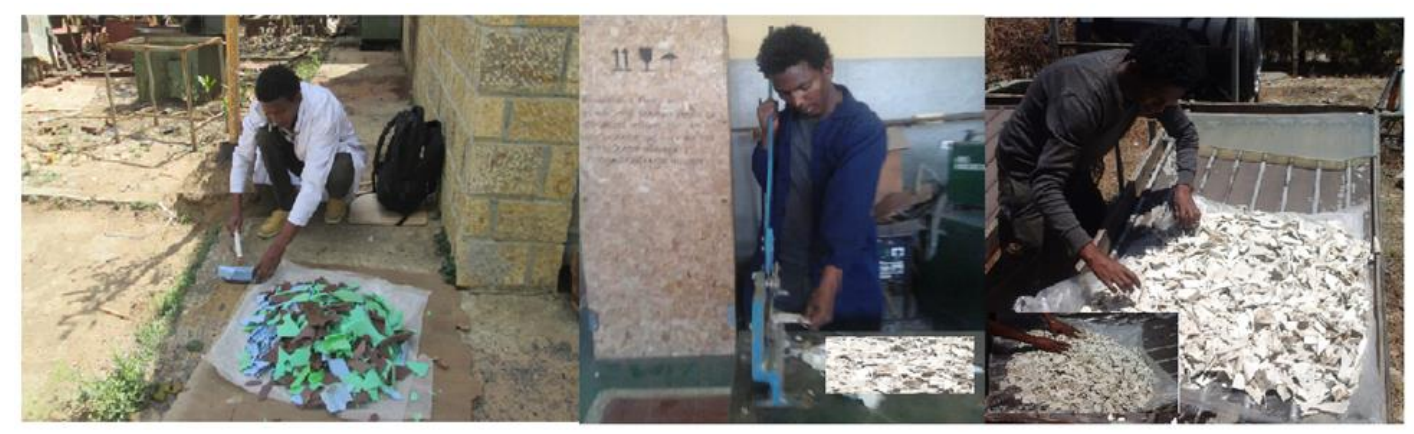

Figure 4. Smithereens of recycled polypropylene

\subsection{Composite preparation}

ASTM D 6641/D 6641M-09 was used as a guideline for the composite fabrication process as depicted in figure 5 and 6.
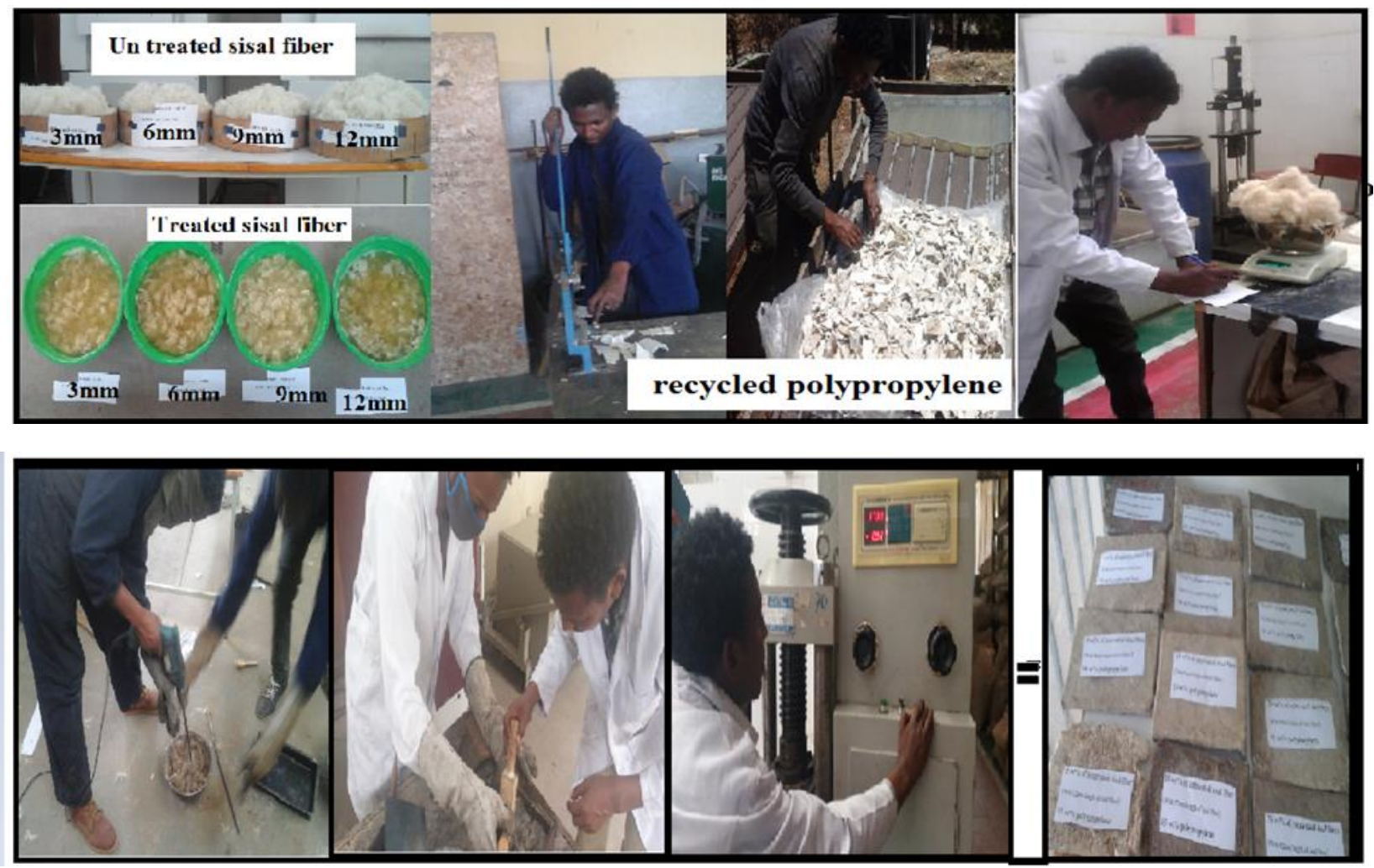

Figure 5. Fabrication procedure for sisal fiber reinforced polypropylene composite

Fiber length, treatment, and loading are the three most important factors taken while preparing the different samples [20]. Fiber orientation was selected to be random to ease the production. Washed recycled polypropylene was put in a stainless steel container then entered to the furnace at $160{ }^{\circ} \mathrm{C}$ [18] for 10 minutes. The liquid waste polypropylene and the treated and untreated sisal fiber with different length and weight ratio (fiber/matrix ratio of 5/95, 15/85, and 25/75) were mixed using portable drill stirrer at a speed of $30 \mathrm{rpm}$, for $15 \mathrm{~min}$ in order to uniformly distribute the fiber with in the matrix. After mixture is allowed to cure for $10 \mathrm{~min}$., compression pressure of $37 \mathrm{MPa}$ (WYA-2000 
Digital Display Hydraulic compression machine) was applied on the mould for 10 minutes, having the uniformly stirred mixture inside. Air gaps formed between the fibers during the processing were gently squeezed out during compression by hydraulic press. Finally the composites were allowed to dry in air at room temperature and get ready for testing.

The samples are coded as follows: ST15/85 = treated fiber with weight ratio of $15 \%$ sisal fiber and $85 \%$ waste poly propylene and SNT15/85 = untreated fiber with weight ratio of $15 \%$ sisal fiber and $85 \%$ waste poly propylene, the other samples are coded in a similar fashion.

\subsection{Testing of Composites}

\subsubsection{Flammability test (ASTM D-635)}

Flammability of sisal fiber reinforced PP composites material was examined by a horizontal burning test according to ASTM D635. The specimen was held horizontally and a flame was applied to one end of the specimen. In this study, a simple ignitability Blue Flame with a temperature of $500^{\circ} \mathrm{C}$ was utilised. The flame was applied at 45 degree to the sample. The sample was exposed to the gas flame for 30s. A burning time from the first reference mark, i.e. $25 \mathrm{~mm}$ from the end, to the second reference mark, i.e. $100 \mathrm{~mm}$ from the end, was recorded. Then, burning rates of the composites were calculated.

\subsubsection{Chemical resistance test}

Chemical resistance test is done to understand the degradation mechanisms induced by the environment and must be studied in order to prevent premature failure of the product.

Water has three major effects when put in contact with composite materials namely, the chemical effect by hydrolysing the unstable bonds; physical effect, destroying the bonds between the materials and photochemical effect. Similarly $\mathrm{NaOH}$ and $\mathrm{H}_{2} \mathrm{SO}_{4}$ have sever effects on composites.

Thus the chemical resistance test of the prepared composite is done for water, sodium hydroxide and sulphuric acid. According to ASTM D 543 testing method, for each of the three chemicals, five pre weighed samples were dipped in the respective chemical reagents (distilled water, $30 \% \mathrm{NaOH}$ solution, $30 \% \mathrm{H}_{2} \mathrm{SO}_{4}$ solution) for $216 \mathrm{~h}$ or for 9 days. The samples are removed and immediately washed in distilled water and dried at oven for $6 \mathrm{hr}$ at $80^{\circ} \mathrm{C}$. Finally the samples are weighed and the percentage weight loss/gain was determined using the formula below [8],

$$
W L(\%)=\frac{W I-W F}{W I} * 100
$$

Where

WL is the weight loss during the immersion/test period; WI is weight of the specimen before immersion and WF is the weight of the specimen after the test period. 


\section{Result and Discussion}

\subsection{Flammability}

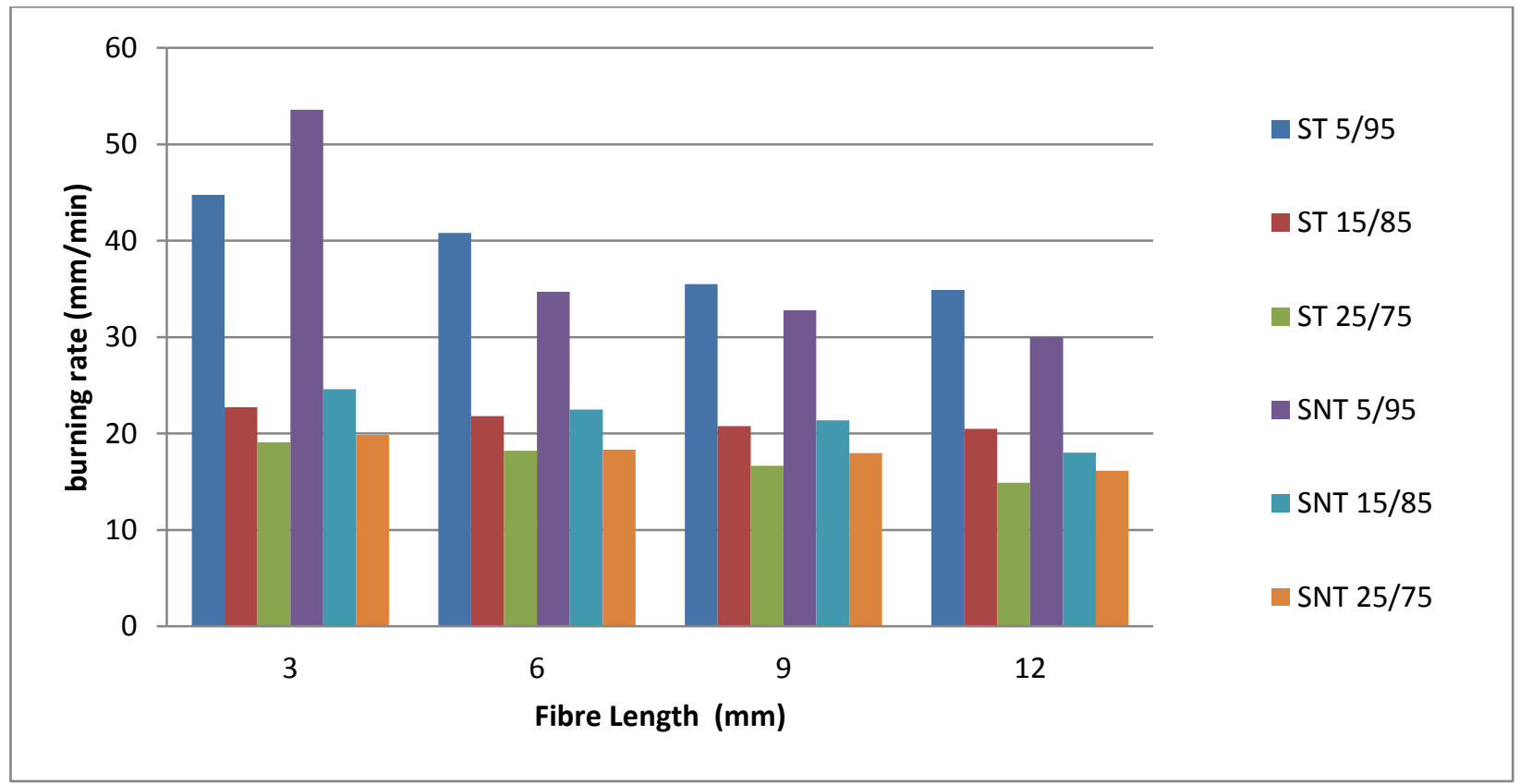

Figure 6. Effect of fiber length, fiber loading and fiber treatment on burning rate

$S T=$ treated fiber; $S N T=$ untreated fiber and 15/85= weight ratio of $15 \%$ sisal fiber and $85 \%$ waste poly propylene

As can be shown in Figure 6, the result indicates that burning rate decreases from 3mm fiber length up to $12 \mathrm{~mm}$ fiber length. This is due to strong bonding formed between fiber and matrix as the length increases.

It is also indicated in figure 6 that treated fiber has lower burning rate $(14.89 \mathrm{~mm} / \mathrm{min})$ than untreated fiber $(16.12 \mathrm{~mm} / \mathrm{min})$. This is because alkali treated SFRPP composite required more oxygen than untreated SFRPP composite to burn and adding flame retardants (4\% NaOH) in to SFRPP composite reduced the burning rate. Generally, adding flame retardants (alkali group) into sisal/PP composites reduces burning rate due to an increase in thermal stability of the composites [17-19].

Effect of fiber/matrix ratio on the flammability in the above figure 6, shows that burning rate decreases as the ratio of fiber to propylene increase from $5 \%$ up to $25 \%$. This might be because as the ratio of fiber to PP increases, strong bond is formed, good interfacial modification created, and high interfacial shear stress between sisal fiber and matrix is maintained [17-19].

\subsection{Resistance to water}

As shown in Figure 7, maximum chemical resistance to water (minimum weight loss) was $0.33 \mathrm{~g}$ followed by $0.36 \mathrm{~g}, 0.68 \mathrm{~g}$ and $0.84 \mathrm{~g}$ for treated fiber to Polypropylene ratio of $5 / 95$ (5\% fiber \& 95\% PP) and $3 \mathrm{~mm}, 6 \mathrm{~mm}, 9 \mathrm{~mm}$, and $12 \mathrm{~mm}$ fiber lengths respectively. This result indicates that chemical resistance property is better at fiber length of $3 \mathrm{~mm}$ than $6 \mathrm{~mm}, 9 \mathrm{~mm}$ and $12 \mathrm{~mm}$. This is because 
shorter fiber is capable to resist diffusion of water molecules than longer one. In addition, short fiber has less pace of water capillary transport in to the gap and flaws at interface between the fiber and matrix than longer fiber [16]. It can be concluded here that composite with a lowest weight loss have low moisture absorption potential.

The other factor that was expected to have an effect was fiber treatment; as a result, treated fibre has shown better chemical resistance to water (minimum weight loss, $0.33 \mathrm{~g}$ ) than untreated fiber $(0.42 \mathrm{~g}$ ) fiber to Polypropylene ratio of 5/95 (5\% fiber \& 95\% PP) and 3mm fiber length. This is because alkali treatment improves strength of the surface of the composite and creates strong adhesion which hinders the hydrophilic behaviour of sisal fiber and reduces water up take behaviour [12-13].

Effect of fiber loading on the chemical resistance for SFRPP composite as shown in Figure 7, indicates that maximum chemical resistance to water (minimum weight loss) is found $0.33 \mathrm{~g}$ at $5 / 95$ fiber to PP ratio followed by $0.56 \mathrm{~g}$ and $0.75 \mathrm{~g}$ for $15 / 85$ and $25 / 75$ fiber to PP ratio respectively for $3 \mathrm{~mm}$ fiber length. This result implies that chemical resistance for fiber to polypropylene ratio of $5 / 95$ (5\% fiber \& $95 \%$ PP) is better than fiber to PP ratio of 15/85 (15\% fiber \& 85\% PP), and 25/75 (25\% fiber \& 75\% PP). This is because, as the fiber content increases the amount of water uptake by the fiber is increased and this leads to reduced resistance to chemicals [21].

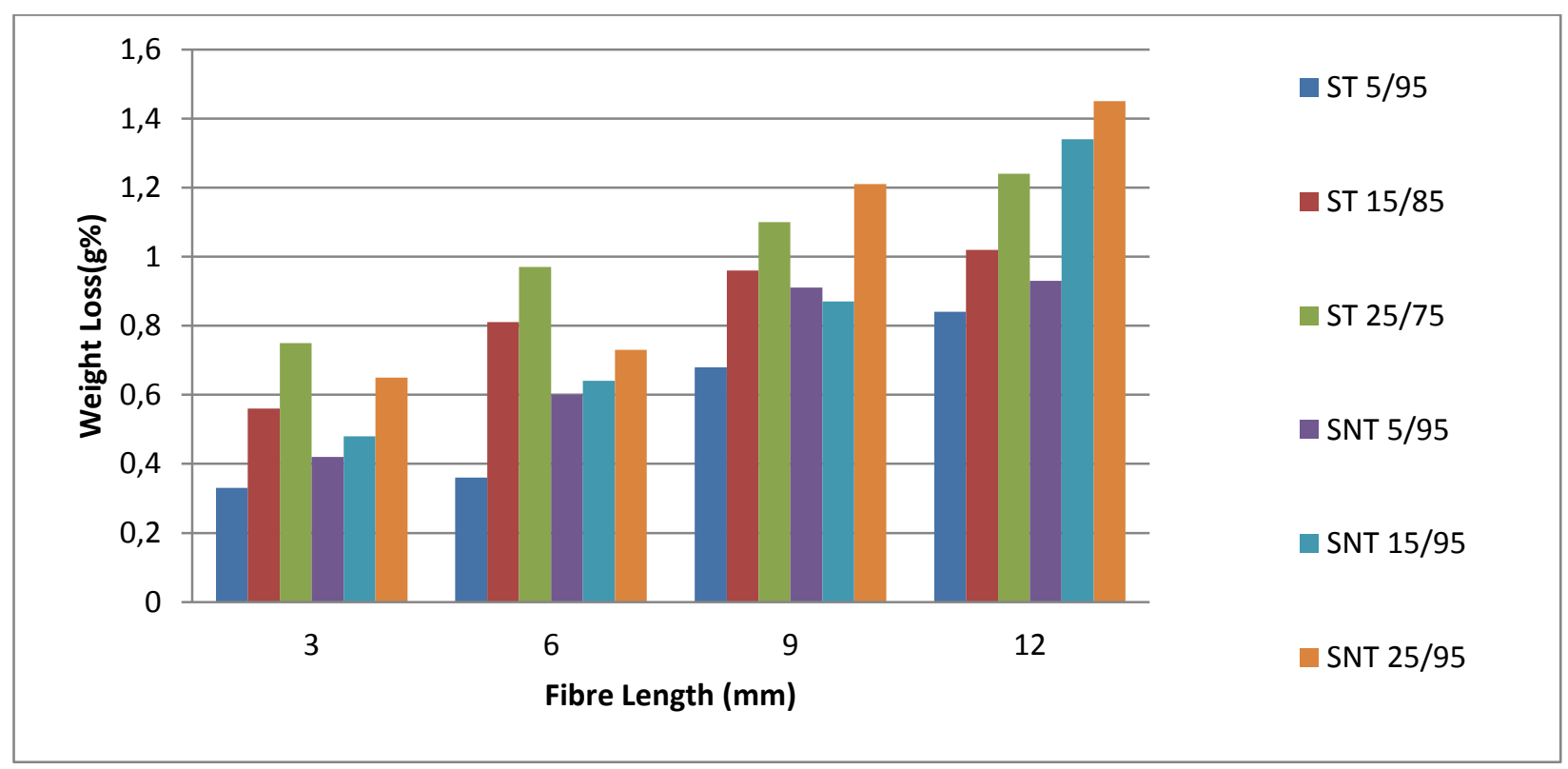

Figure 7. Resistance of the SFRPP composites to water

$S T=$ treated fiber; $S N T=$ untreated fiber and 15/85= weight ratio of 15\% sisal fiber and $85 \%$ waste poly propylene

\subsection{Resistance to sodium hydroxide $(\mathrm{NaOH})$}

Observing the results displayed in figure 8 below, it is easy to analyse that fibre loading has a significant effect on resistance to $\mathrm{NaOH}$; the results for fiber loading lays between 1.3-1.4g weight loss for 5\%, 15\% fiber loading at all the fiber lengths tested and for both treated and untreated fiber. On the contrary, as the fiber loading increases to $25 \%$ by weight, the weight loss range has increased to 2.3-2.4.g. This might be due to the susceptibility of sisal fibre to alkaline chemicals. On the other hand, Fibre length and treatment were found to have less significant when compared with fiber loading. 


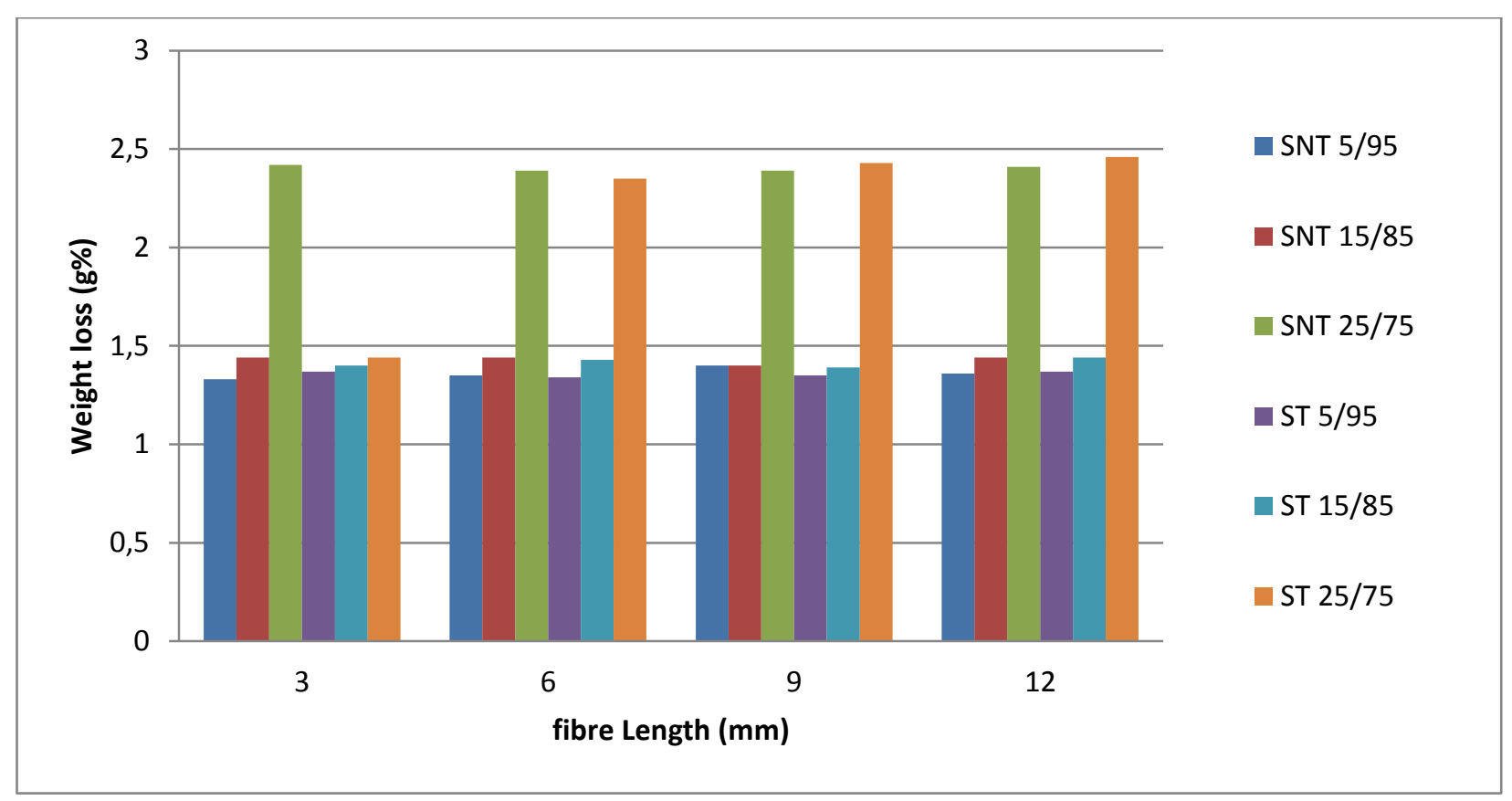

Figure 8, Resistance of the composites to $30 \% \mathrm{NaOH}$

$S T=$ treated fiber; $S N T=$ untreated fiber and 15/85= weight ratio of 15\% sisal fiber and $85 \%$ waste poly propylene

\subsection{Resistance to sulphuric acid $\left(\mathrm{H}_{2} \mathrm{SO}_{4}\right)$}

As can be seen in figure 9 below, the fiber loading displays a significant effect on the percentage weight loss. The SFRPP composite with a $12 \mathrm{~mm}$ treated sisal fiber length at the last right side of the picture displays a weight loss $1.4 \%, 1.8 \%$, and $2.8 \%$ as the fiber loading increases from $5 \%$, to $15 \%$ and to $25 \%$ respectively. For a constant fiber length and treatment, the same trend (an increase in weight loss) is observed for an increase in fiber loading throughout the picture. The results lead to a conclusion that the amount fiber in the composites has greatly influenced the resistance to $\mathrm{H}_{2} \mathrm{SO}_{4}$. Figure 9 also depicts that the weight loss for the 25\% treated sisal fiber loaded composite material displays a result of above $2.5 \%$. But, weight loss of the $25 \%$ treated fiber loaded SFRPP is less than $2.5 \%$ except for the $12 \mathrm{~mm}$ long sisal fiber. Similar trend is also observed for the other fiber loadings. This is attributed to the sense that fiber treatment has a significant effect on the resistance of the composite to $\mathrm{H}_{2} \mathrm{SO}_{4}$. The parameter that has shown less significant is the variation in fiber length. Generally, weight loss is highly dependent on fibre loading followed by treatment and fibre length. 


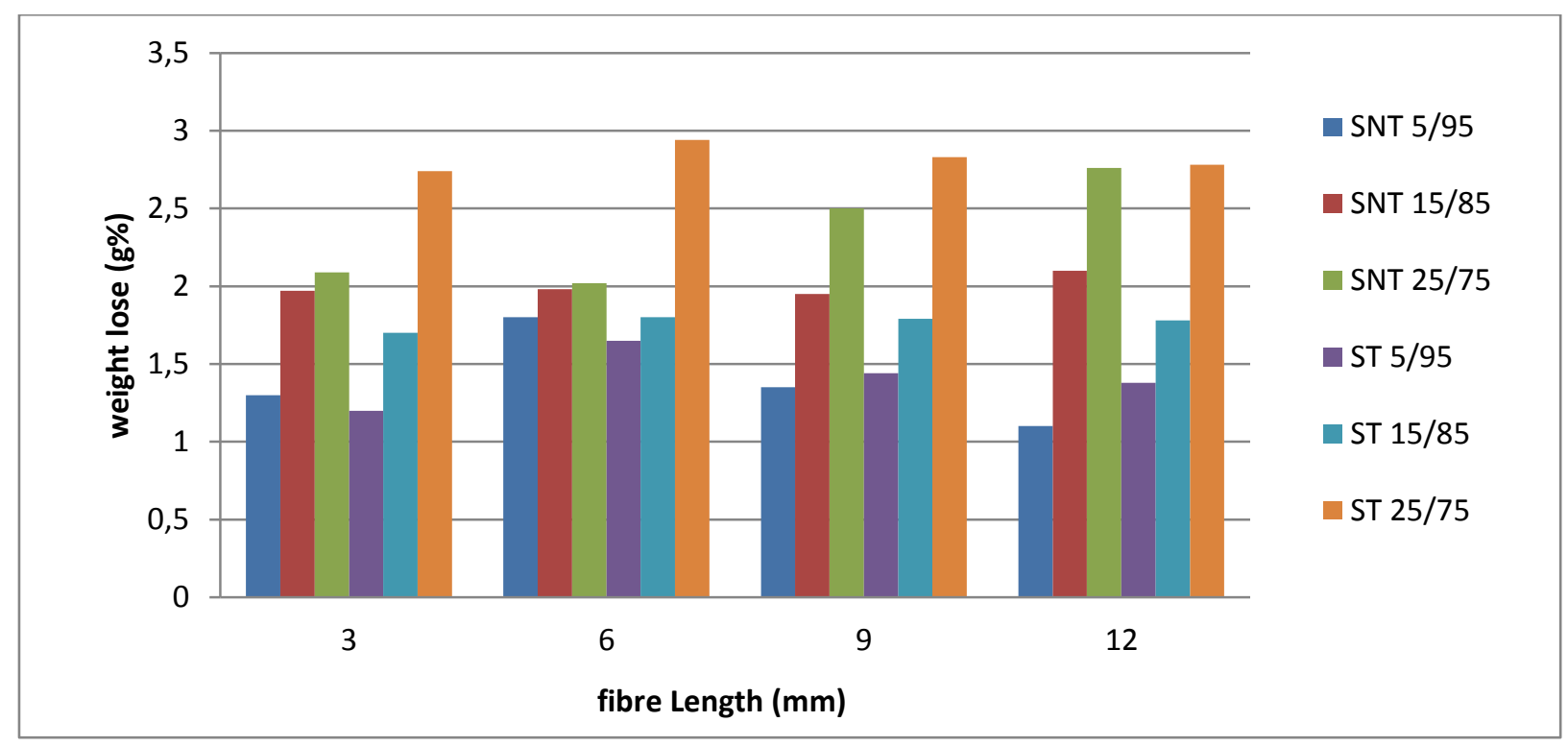

Figure 9, Resistance of the composites to $30 \% \mathrm{H}_{2} \mathrm{SO}_{4}$

$S T=$ treated fiber; SNT = untreated fiber and 15/85= weight ratio of 15\% sisal fiber and 85\% waste poly propylene

\section{Conclusion}

This research was initiated to develop car internal door trim from waste polypropylene composed with sisal fiber. Taking the fiber loading, fiber length and fiber treatment as critical parameters, the flammability and chemical resistance property of the composites is evaluated. With SFRPP composite is found to have better resistance to water than $\mathrm{NaOH}$ and $\mathrm{H}_{2} \mathrm{SO}_{4}$ and treating the fibre has brought considerable improvement on chemical resistance of the composite. Fibre loading and fibre length has positive and negative effect on the flammability of the SFRPP composite respectively. Improvement on flammability has come with a disadvantage on chemical resistance, Treated sisal fibre with 15\%:85\% fibre to polypropylene ratio at $9 \mathrm{~mm}$ fibre length seems to be an optimal product for car interior door trim.

\section{References}

[1] Mwaikambo L. Y. (2006) Review of the history, properties and application of plant fibres. African journal of science and technology (AJST), science and engineering series. 7 (2) pp. 120-133.

[2] M. Hautala M. - Pasila A. - Pirila J. (2004) Use of Hemp and Flax in Composite Manufacture: A Search for new Production Methods. Composite Part A. www.elsevier.com/locate/composites. 35, pp. 11-16.

[3] Brahmakumar M. - Pavithran C. - Pillai R.M. (2005) Coconut Fibre Reinforced Polyethylene Composites: effect of Natural Waxy Surface Layer of the Fibre on Fibre/matrix Interfacial bonding and Strength of Composites. Material Processing Technology. 65(3-4), pp. 563-569.

[4] Oladele I. O. - Adewuyi B.O. (2008) Development of Automobile Gaskets from Local Fibres. Journal of Science and Technology. 28(3) pp. 152-157.

[5] Malkapuram R. - Kumar V. - Yuvraj S.N. (2008) Recent Development in Natural Fibre Reinforced Polypropylene Composites. Journal of Reinforced Plastics and Composites. 28 pp. 1169-1189. 
[6] D. Fengel - G. Wegener (1984) Wood: Chemistry, Ultrastructure, Reactions. Journal of Polymer Sciences Walter de Gruyter. Berlin. 482.

[7] Beige Right Inner Door Panel Handle Pull Trim Cover Fit BMW E70 X5 51416969404, https://www.ebay.com/sch/Car-Truck-Interior-Door-Panels-Parts-for-Peugeot

[8] Livonia, MI (1990) Safety and Technical Rescue Association.

[9] N. Suppakarn - K. Jarukumjorn (2009) Mechanical properties and flammability of sisal/PP composites: Effect of flame retardant type and content. Composites: Part B 40. pp. 613-618.

[10] E.S. Rodriguez - P.M. Stefani - A. Vazquez (2007) Effects of fibers' alkali treatment on the resin transfer molding processing and mechanical properties of Jute-Vinylester composites. Journal of Composite Materials. 41(14) pp. 1729-1741.

[11] R.N. Turukmane - A.L.Bhongade - S.P.Borkar - A.M. Daberao (2017) Studies on Inter fibre cohesion Properties of Sisal Fibre reinforced Polypropylene Composite. Studies. 3 (1)

[12] J. Rout - M. Misra - S.S. Tripathy - S.K. Nayak - A.K. Mohanty (2001) The influence of fibre treatment on the performance of coir-polyester composite. Compos Sci Technol. 61 pp. 13031310.

[13] I.V. Weyenberg - T.C. Truong - B. Vangrimde - I. Verpoest (2006) Improving the properties of UD flax fibre reinforced composites by applying an alkaline fibre treatment. Compos A: Appl Sci Manuf. 37 pp. 1368-1376.

[14] W. Liu - A.K. Mohanty - P. Askeland - L.T. Drzal - M. Misra (2004) Effects of alkali treatment on the structure, morphology and thermal properties of native grass fibers as reinforcements for polymer matrix composites. J Mater Sci. 39 pp. 1051-1054.

[15] Boopathi L. - Sampath P.S. - Mylsamy K. (2012) Investigation of physical, chemical and mechanical properties of raw and alkali treated Borassus fruit fiber, Composites. Part B. 43 pp. 3044-3052.

[16] Ayrilmis, N. - Jarusombuti, S. - Fueangvivat, V. - Bauchongkol, P. - White, R. H. (2011). Coir fiber reinforced polypropylene composite panel for automotive interior applications. Fibers and Polymers, 12(7), 919.

[17] I. Musanif - A. Thomas (2015). Effect of Alkali Treatments of Physical and Mechanical Properties of Coir Fiber. Chemical and Materials Engineering 3(2) pp. 23-28.

[18] Bharath, K. N. - S. Basavarajappa. (2014) Flammability Characteristics of Chemical Treated Woven Natural Fabric Reinforced Phenol Formaldehyde Composites. Procedia Materials Science. 5 pp. 1880-1886.

[19] Sumaila, M. - I. Amber, - M. Bawa (2013) Effect of fiber length on the physical and mechanical properties of random oriented, nonwoven short banana (musa balbisiana) fibre/epoxy composite. Cellulose. 62 p. 64.

[20] Razak, J. A. - Akil, H. M. - Ong, H. (2007) Effect of inorganic fillers on the flammability behavior of polypropylene composites. Journal of Thermoplastic Composite Materials. 20(2) pp. 195-205.

[21] Girisha, C. - Gunti Sanjeevamurthy - G. R. Srinivas (2014) Sisal/coconut coir natural fibers-epoxy composites: water absorption and mechanical properties. International Journal of Engineering and Innovative Technology (IJEIT) 2.3 pp. 166-170.

[22] Muktha, K. - Gowda, B. K. (2017) Investigation of Water Absorption and Fire Resistance of Untreated Banana Fibre Reinforced Polyester Composites. Materials Today: Proceedings. 4(8) pp. 8307-8312. 
[23] Chow, C. P. L. - Xing, X. S. - Li, R. K. Y. (2007) Moisture absorption studies of sisal fibre reinforced polypropylene composites. Composites Science and Technology. 67(2) pp. 306-313.

[24] A. Lakshumu Naidu - P. S. V. Ramana Rao (2016) A review on chemical behavior of natural fiber composites. Int. J. Chem. Sci. 14(4) pp. 2223-2238.

[25] Joseph, P.V. - Rabello, M.S. - Mattoso, L.H.C. - Joseph, K. - Thomas, S. (2013) Environmental effects on the degradation behaviour of sisal fibre reinforced polypropylene composites. Compos. Sci. Technol. 62 pp. 1357-1372.

[26] Boopathi L. - Sampath P.S. - Mylsamy K. (2012) Investigation of physical, chemical and mechanical properties of raw and alkali treated Borassus fruit fiber. Composites. Part B. 43. pp. 3044-3052.

[27] Oushabi, A. - Sair, S. - Hassani, F. O. - Abboud, Y. - Tanane, O. - El Bouari, A. (2017) The effect of alkali treatment on mechanical, morphological and thermal properties of date palm fibers (DPFs): study of the interface of DPF-Polyurethane composite. South African Journal of Chemical Engineering. 23 pp. 116-123.

[28] Farida, Y. - Salawudeen, T. O. - Qasim, H. S. - Ma'an F. A. - Suleyman, A. M. (2011) Chemical resistance tests on PP-ternary nanocomposite for its application in bioreactor liner fabrication. Science China Technological Sciences. 54(8) pp. 2217-2223. 